\title{
Cognitive Sequelae of Traumatic Brain Injury
}

\author{
Amanda R. Rabinowitz, Ph.D. ${ }^{1,2}$ and Harvey S. Levin, Ph. D. ${ }^{3}$ \\ ${ }^{1}$ University of Pennsylvania School of Medicine, Michael E. DeBakey Veterans Affairs Medical \\ Center
}

${ }^{3}$ Baylor College of Medicine and Michael E. DeBakey Veterans Affairs Medical Center

\section{Keywords}

Traumatic Brain Injury; mTBI; Concussion; Executive Functioning; Neuropsychological Assessment

\section{Introduction}

Traumatic brain injury (TBI) has a profound effect on injured individuals and their families. Cognitive deficits caused by TBI interfere with work, relationships, leisure, and activities of daily living, exacting a personal and economic cost that is difficult to quantify.

The cognitive sequelae of TBI are determined by a number of injury related variables including TBI severity, complications, concomitant injuries to other body regions, and chronicity of the injury. Patient characteristics such as age, pre-injury neuropsychiatric status, and genotype also play a role. In addition, cognitive recovery from TBI can also be moderated by the quality of the post-acute environment In this paper we review the cognitive sequelae of closed head injury in adults, with a focus on deficits of executive function and everyday decision-making. Although animal models have made valuable contributions to knowledge of TBI, we focus on human studies for the sake of clinical relevance. First, we review the epidemiology and nature of cognitive changes following TBI. We will also discuss the pathophysiology of TBI-related cognitive deficits, and review clinical assessment and treatment options.

\section{Epidemiology of Cognitive Dysfunction Following TBI}

\section{Short Term Cognitive Impairment}

Impaired consciousness and post-traumatic amnesia (PTA) are neurobehavioral hallmarks of acute TBI. According to consensus definitions, moderate and severe TBI are characterized by loss of consciousness for greater than 30 minutes and/or PTA persisting for at least 24 hours. PTA is the transitory period of disorientation, confusion, and amnestic memory impairment following TBI, which leaves a gap in memory. Patients in PTA also commonly experience disturbed sleep-wake cycle, decreased daytime arousal, agitation, affective

\footnotetext{
(C) 2013 Elsevier Inc. All rights reserved

${ }^{2}$ Please send correspondence to this author Department of Neurosurgery University of Pennsylvania School of Medicine 370 Stemmler Hall Philadelphia, Pennsylvania 19104 Phone: 732-213-8899 rabinowitz.a@ gmail.com.
}

Publisher's Disclaimer: This is a PDF file of an unedited manuscript that has been accepted for publication. As a service to our customers we are providing this early version of the manuscript. The manuscript will undergo copyediting, typesetting, and review of the resulting proof before it is published in its final citable form. Please note that during the production process errors may be discovered which could affect the content, and all legal disclaimers that apply to the journal pertain. 
lability, and perceptual disturbance (6). While in PTA gross fluctuations in the severity of cognitive and behavioral symptoms are common. During this acute confusional state, patients require supervision at the mild end of the spectrum and hospitalization at the moderate to severe end of the spectrum. Contrastingly, mTBI may occur with or without loss of consciousness and PTA.

Recovery on clinical outcome measures typically occurs within several days after uncomplicated sports-related mTBI in young adults, but the clinical course is typically longer in patients with other injury etiologies (e.g., motor vehicle crashes), pre-injury risk factors, concomitant injuries to other body regions, or age older than 50 years. In general, cognitive deficits associated with mild TBI (mTBI) resolve fully within three to six months in about 80-85 percent of patients who sustain their first mTBI without the presence of a brain lesion on computed tomography or other intracranial complication (7). Although there appears to be a subgroup of mTBI patients with persistent cognitive complaints $(2,3)$, the precise prevalence and etiology of these sequelae remain unclear. Data from a recent, prospective cohort study suggests that approximately one third of mTBI patients continue to experience functional impairment (Glasgow Outcome Scale-Extended score $\leq 6$ ) three months post-injury (8).

Moderate and severe TBI are associated with more severe and persistent cognitive deficits, which can involve cognitive domains typically preserved in mild TBI, such as awareness, reasoning, language, visuospatial processing, and general intelligence.

\section{Long Term Cognitive Impairment}

As many as $65 \%$ of moderate to severe TBI patients report long-term problems with cognitive functioning (1). Cognitive deficits cause disability when they interfere with a patient's ability to perform their usual roles at work or at home. Epidemiologic research indicates that about $43 \%$ of these patients experience disability over periods of six months or longer, characterized by functional limitations, post-injury symptoms that limit activities, cognitive complaints, and/or mental health problems (4). Nearly a quarter of moderate to severe TBI patients fail to return to work in the year following their injury (1). At their most extreme, TBI-related cognitive deficits can impair a patient's ability to perform activities of daily living, such as driving, meal preparation, and handling money. Although TBI can cause sensory and motor deficits, cognitive and behavioral changes are more closely associated with long-term disability (9).

In general, the relationship between acute TBI severity and cognitive sequelae is approximately linear with longer duration of impaired consciousness predicting greater extent of cognitive dysfunction (10). However, heterogeneity in TBI pathology and patient characteristics also influence cognitive outcomes, complicating prediction of recovery. The cognitive domains that are most often affected by mild to moderate TBI include memory, attention, processing speed, and executive functioning and are mostly resolved within 3-6 months of injury $(7,11-13)$. However, it is possible that cognitive dysfunction in a subgroup of individuals is poorly characterized by effect-size estimates based on aggregate data (14). Moderate to severe TBI is also associated with deficits in memory, attention, processing speed, and executive functioning, and additionally involves functions such as communication, visuospatial processing, intellectual ability, and awareness of deficit (1517).

Cognitive recovery after moderate to severe TBI has a steep trajectory in the first year followed by more gradual improvements during later years. Impairments are more likely to persist in patients with severe injuries; although some patients may exhibit neuropsychological recovery up to 5 years post injury (18). Gains in cognitive performance 
after the first year are likely to be a function of new learning and development of compensatory strategies (18). A summary of the acute and long-term cognitive sequelae of TBI is provided in Table 1.

\section{Deficits of Executive Function and Everyday Thinking Skills}

Executive functioning deficits are common following TBI, even among those with mild injuries. The term "executive function" describes a variety of high order cognitive abilities predominantly subserved by regions in the prefrontal cortex and their circuitry. Deficits of the executive system threaten an individual's ability to engage successfully in independent goal-oriented behavior. Hence, these functions are critically important for quality of life, as they are implicated in job performance, social relationships, and both basic and instrumental activities of daily living (19). Results of a recent study suggest that executive deficits are more predictive of functional disability than demographic and injury variables (20). Both cognitive and behavioral functions fall under the general umbrella of executive functioning, and many of these can be affected by TBI.

- Cognitive Executive Functions

$\begin{array}{ll}\bigcirc & \text { Memory Acquisition and Retrieval } \\ \bigcirc & \text { Top-down Control of Attention } \\ \bigcirc & \text { Planning } \\ \bigcirc & \text { Judgment } \\ \bigcirc & \text { Cognitive Aspects Decision-Making } \\ \bigcirc & \text { Emotional Aspects of Decision-Making } \\ \bigcirc & \text { Motivation } \\ \bigcirc & \text { Impulsivity }\end{array}$

Various authors have conceptualized executive functions in a number of different ways. Characterizations tend to share in common the notion that the executive system involves distinct components, which are, at least to some extent, functionally and anatomically dissociable. Within an individual patient any number of these functional domains may be affected or spared. Nearly all characterizations of executive function include components describing the control and direction of lower level cognitive abilities, motivation, and selfmonitoring. These functions influence many everyday cognitive tasks, including learning and memory, planning, decision-making, and social behavior $(19,21)$.

Executive dysfunction can lead to apparent disruption of cognitive performance in other related domains, such as memory and top-down control of attention. Memory problems are a common complaint following TBI, but the nature of TBI related memory difficulties is quite different from what is typically seen in Alzheimer's Disease or other amnestic memory disorders. Unlike an amnestic memory disorder, memory problems in TBI are not typically due to a deficit of memory storage-that is, TBI patients generally retain the ability to recognize newly learned material. Rather, TBI patients tend to have difficulties organizing new information for successful encoding and retrieval $(22,23)$. Because of disorganized memory encoding, TBI patients are more likely to attribute information to the wrong source, e.g. a patient might misremember that a piece of information was told to him by his wife, when it was in fact relayed by his doctor. They are also more likely to conflate different pieces of information e.g., a patient might mistakenly conflate the appointment date for a doctor's appointment with the appointment time for a salon appointment. 
Planning abilities are also depressed following TBI. Effective planning depends on an individual's ability to hold rules and conditions in mind using working memory, represent and keep track of available options, and forecast the consequences of each course of action. These skills are typically evaluated using tests that require the examinee to achieve a goal as efficiently as possible, while following a set of rules. TBI patients are more likely than uninjured individuals to violate the rules and take unnecessary steps in their execution of a task (24). These types of difficulties translate to real-life problems, such as inefficiencies and errors on the job or at home. Instrumental activities of daily living, such as cooking, shopping, driving, and finance management can be affected.

Judgment and decision-making may also be impaired in moderate-to-severe TBI; however, these deficits are rare at the mild end of the TBI severity spectrum (25). Human reasoning depends on both effortful cognitive operations and the automatic input from visceral or emotional states (26). The somatic marker hypothesis has been put forth to explain the role of emotion in decision-making. Decision-making typically involves the selection of an option among a set of choices (decision space). According to the somatic marker hypothesis, humans associate visceral and emotional states with each item in the decision space according to their prior experience with those options. Options associated with reward are emphasized, whereas those associated with negative outcomes are suppressed. These emotional inputs are integrated with rational analysis to produce a decision (27). Changes in the neural mechanisms underlying visceral responses and their associations with positive and negative outcomes can impair an individual's ability to optimally adjust their behavior in the face of risks. Patients with moderate to severe TBI may exhibit deficits in the various aspects of decision-making, such as impulsivity, risk adjustment, and rational choice. These impairments are related to neuroimaging abnormalities in the anatomical regions associated with each of those functions (28).

The diminished capacity to initiate activity can be one of the most devastating consequences of a brain injury. Apathy is a condition characterized by reduced goal-directed activity and lowered motivation. Point-prevalence rates of apathy in severe TBI patients range from $46 \%$ to $71 \%$ (29). This is a serious problem, as it can lead to social withdrawal and neglect of important self-care activities. Across neurological diseases, apathy is associated with a number of adverse outcomes, including decreased functional level, caregiver distress, and poor treatment response (27). It is unclear whether apathy is the cause of these deleterious outcomes, or if apathy and negative outcomes are associated via a lurking variable, such as injury severity. Deficits in motivation and drive are more prevalent in severe TBI with focal lesions involving the right frontal lobe (27).

Deficits of self-awareness and self-monitoring are also associated with severe TBI. As might be expected, these problems pose a serious challenge for rehabilitation and treatment. At its most severe, patients exhibit anosognosia, or the failure to recognize the existence of a disease or injury (30). Impaired awareness can take on many forms in TBI patients. Acutely, patients may be unaware that they have suffered an injury at all. Later in the clinical course, patients may be aware of their injury, but underestimate its impact on their ability to function (31). Patients tend to have better awareness for physical (e.g., hemiparesis) rather than cognitive deficits (31).

Metacognition refers to the conscious awareness of one's cognitive abilities (32). Metacognitive knowledge describes patients' general awareness of their cognitive abilities, which can be measured by evaluating the discrepancy between self-report of cognitive problems and actual neuropsychological performance. Emergent and anticipatory awareness during the execution of a cognitive task represent a different category of metacognitive skills. The ability to notice and respond to errors is an emergent form of awareness that 
allows an individual to modulate their approach to a task in order to optimize success. The ability to predict future performance is an anticipatory metacognitive skill that allows an individual to forecast their success and prepare for optimal performance (33). All three metacognitive skills can be affected by TBI.

\section{Pathophysiology of Cognitive Deficits Following TBI}

Closed head TBI occurs when the brain undergoes rapid acceleration-deceleration forces, which may involve blunt head impacts. However, rapid acceleration or deceleration of the head can occur without impact. The resultant biomechanical forces can include both translational (the head moving in a straight line with the brain's center of gravity) and rotational (the brain rotating around its center of gravity) accelerations. Although there is great heterogeneity in the biomechanical profile of TBI, there is a predominant injury profile that suggests the vulnerability of specific gray matter regions and white matter pathways. Contusions, or focal damage to the brain's tissue and vasculature structure, are most likely to occur in frontal and temporal regions, where brain tissue is adjacent to the bony ridges and protuberances of the interior base of the skull (34). Intracranial hemorrhage may occur, and represents an acute medical emergency. Diffuse axonal injury (DAI) refers to damage of the brain's white matter tracts, caused by sheering forces that result when the brain rotates within the skull $(35,36)$. Due to their viscoelastic properties, axonal damage is prevalent across levels of injury severity, and DAI is likely to be the principal pathological substrate of long-term deficits associated with mTBI $(37,38)$.

Given the high prevalence of executive deficits in TBI patients, it is not surprising that the frontal lobes and their related circuitry (e.g. subcortical white matter, basal ganglia, and thalamus) are particularly vulnerable to TBI (5). Working memory and planning deficits may be associated with the focal injury to the dorsolateral prefrontal cortex (DLPFC) or DAI affecting the projections between the lateral frontal and posterior regions (39). Apathy has been associated with subcortical lesions and right hemisphere dysfunction (40). Impaired awareness is characteristic of patients with focal frontal injury (41), and the postinjury level of self-referential insight has been associated with the integrity of right dorsal prefrontal cortex (42).

Decision-making is a complex cognitive function, and Newcombe and colleagues (2011) examined the diffusion tensor imaging (DTI) correlates of its component skills in patients with moderate to severe TBI. Deficits in risk adjustment were associated with abnormalities in subcortical structures such as the thalamus, the dorsal striatum and the caudate. Impulsivity was associated with abnormal DTI findings in the bilateral orbital frontal gyri, insula, and caudate; whereas impaired rational choice was related to changes in the bilateral DLPFC, the superior frontal gyri, and the right and ventromedial prefrontal cortex, ventral striatum, and hippocampus (28). This pattern of results suggests that the emotional components of decision making, i.e. risk adjustment and impulse control, predominantly involve subcortical structures and the interplay between frontal and subcortical systems; whereas cognitive components of decision-making, such as rational choice, rely heavily on the prefrontal cortex.

Focal structural abnormalities are infrequent on computed tomography within 24 hours after mTBI. However, advanced neuroimaging techniques have begun to elucidate the effects of mild brain injury. DTI is a neuroimaging technique that measures the diffusion of water in tissues, which has been used to evaluate the integrity of the brain's white matter. DTI studies of mTBI patients corroborate findings from patients with more severe injuries, suggesting that frontal and temporal pathways are most vulnerable to traumatic damage $(38,43-45)$. 


\section{Neuropsychological Assessment}

Cognitive functioning should be assessed in any patient with TBI. Interviewing the patient and a caregiver is recommended because a subgroup of patients with severe TBI has poor awareness of the presence or extent of their cognitive deficits and behavioral sequelae. An interview can evaluate the accuracy of the patient's insight concerning their condition by asking them to appraise their memory for events and names of people from one day to another, plans for return to work or school, organization of activities, and relations with family members, colleagues, and friends. Patients' self-reported problems provide the clinician with information regarding the deficits causing greatest subjective distress. Behavioral observations during an interview can also provide valuable information regarding the patient's sustained concentration, ability to shift attention from one task to another, and vulnerability to distraction. Comparison of the patient's report with input from a relative or caregiver is informative especially if the collateral source is interviewed separately from the patient.

To harmonize research across centers and facilitate comparison of clinical data obtained at different sites, the Federal Interagency Traumatic Brain Injury Research (FITBIR) Task Force, including the National Institutes of Health (NIH), has published a list of recommended outcome measures for clinical TBI research, referred to as Common Data Elements (CDEs). The CDEs include core, basic, and supplemental cognitive tests commonly used in clinical practice $(46,47)$.

Referral to a clinical neuropsychologist provides a more in-depth evaluation using tests which have been developed to be reliable and valid measures of cognition, behavior, and self-reported symptoms. With reference data obtained from large samples of representative subjects in the general population, the results of neuropsychological assessment can be reported in percentiles or standard scores that reflect the severity of deficits and relatively preserved abilities. Clinical neuropsychologists could also recommend further rehabilitation and other treatment that is implicated by the pattern of findings. Table 2 lists several of the tests that are widely used by neuropsychologists to assess memory, attention, processing speed and executive function. The "CDE" designation in Table 2 denotes that it was recommended by panels of neuropsychologists as a Common Data Element $(46,47)$. The CDE outcome measures have been used extensively in research on recovery from TBI; the reliability and validity of these measures are also established.

There is an extensive body of literature supporting the sensitivity of neuropsychological tests to TBI related cognitive deficits $(7,11,13,48)$. Typical neuropsychological assessment of TBI includes tests of episodic memory, attention, cognitive processing speed, and executive functions, such as mental flexibility, planning, decision-making, inhibitory control, and organization. Assessment of mild TBI should also include a measure of postconcussion symptoms. For patients with moderate to severe injuries, awareness and judgment should be evaluated in order to determine the patient's capacity to function independently. The results of a neuropsychological assessment provide information regarding a patient's cognitive areas of weakness and preserved strengths. Findings can be particularly useful when there are questions regarding occupational and education planning. A neuropsychologist may also recommend strategies to help the patient compensate for deficits.

\section{Treatment}

Rehabilitation is a broad health care field primarily concerned with reducing patients' disability and enabling their independent functioning following a disease or injury. Rehabilitation for a physical disability, such as paralysis, might include physical exercises, 
assistive technology, skill training, as well as social services. Cognitive rehabilitation therapy (CRT) is a term that describes treatments designed to improve patients' participation in cognitive demanding activities, either by restoring cognitive functions or teaching compensatory skills. Like physical rehabilitation treatments, CRTs might incorporate technologies and services that facilitate patients' reintegration into their pre-injury lifestyle.

Specific CRTs have been designed to address problems with attention, communication, memory, and executive functioning. Goal Management Training (GMT) is an example of a CRT for the treatment of executive functioning deficits based on theories of goal processing and sustained attention. Its primary objective is to train patients to periodically pause their ongoing behavior to monitor performance and define goal hierarchies. Cues, such as audible tones, can serve as reminders to stop and monitor behavior. A recent controlled study showed that brain injured patients who received GMT showed evidence of cognitive improvements on tests of attention and planning (49). The Institute of Medicine (IOM) recently assembled a Committee on CRTs for TBI. The committee reviewed the evidence in support of CRTs across levels of injury, phases of recovery, and cognitive domains. They noted methodological limitations in the extant research literature on CRTs, but nonetheless, supported the ongoing use of CRT for ameliorating the cognitive sequelae of TBI (50). Future studies should employ randomized controlled designs to best evaluate existing CRTs and identify which patients stand to benefit most from these intervention.

\section{Summary}

Cognitive deficits are common following TBI and contribute significantly to disability. The frontal lobes and their related circuitry are particularly vulnerable to traumatic damage; hence executive dysfunction is prevalent. Impairments in executive dysfunction can profoundly impact patients' quality of life, as these cognitive skills are implicated in job performance, social relationships, and activities of daily living. A neuropsychological evaluation provides a comprehensive assessment of patients' cognitive strengths and weaknesses. Cognitive rehabilitation is an appropriate treatment option for TBI patients with cognitive deficits; however, more methodologically rigorous research is needed to demonstrate its efficacy and identify which patients are most likely to respond.

\section{Acknowledgments}

This research was supported by Mild Traumatic Brain Injury and Diffuse Axonal Injury. NINDS Grant P01 NS056202 awarded to Douglas Smith, M.D. and Neurobehavioral Outcome of Head Injury in Children. NINDS Grant NS-21889 awarded to Gerri Hanten, Ph.D. and Harvey Levin, Ph.D.

\section{Acronyms}

$\begin{array}{ll}\text { TBI } & \text { Traumatic brain injury } \\ \text { PTA } & \text { post-traumatic amnesia } \\ \text { mTBI } & \text { Mild traumatic brain injury } \\ \text { DAI } & \text { Diffuse axonal injury } \\ \text { DLPFC } & \text { dorsolateral prefrontal cortex } \\ \text { DTI } & \text { diffusion tensor imaging } \\ \text { FITBIR } & \text { Federal Interagency Traumatic Brain Injury Research } \\ \text { NIH } & \text { National Institutes of Health } \\ \text { CDEs } & \text { Common data elements }\end{array}$




$\begin{array}{ll}\text { CRT } & \text { Cognitive rehabilitation therapy } \\ \text { GMT } & \text { Goal Management Training } \\ \text { IOM } & \text { Institute of Medicine }\end{array}$

\section{References}

1. Whiteneck GG, Gerhart KA, Cusick CP. Identifying environmental factors that influence the outcomes of people with traumatic brain injury. The Journal of Head Trauma Rehabilitation. 2004; 19(3):191-204. [PubMed: 15247842]

2. Boake C, McCauley SR, Levin HS, et al. Diagnostic criteria for postconcussional syndrome after mild to moderate traumatic brain injury. The Journal of Neuropsychiatry and Clinical Neurosciences. 2005; 17(3):350-6. [PubMed: 16179657]

3. Røe C, Sveen U, Alvsåker K, Bautz-Holter E. Post-concussion symptoms after mild traumatic brain injury: influence of demographic factors and injury severity in a 1-year cohort study. Disability \& Rehabilitation. 2009; 31(15):1235-43. [PubMed: 19116810]

4. Selassie AW, Zaloshnja E, Langlois JA, et al. Incidence of long-term disability following traumatic brain injury hospitalization, United States, 2003. The Journal of Head Trauma Rehabilitation. 2008; 23(2):123-31. [PubMed: 18362766]

5. MacDonald BC, Flashman LA, Saykin AJ. Executive dysfunction following traumatic brain injury: Neural substrates and treatment strategies. NeuroRehabilitation. 2002; 17:333-44. [PubMed: 12547981]

6. Nakase-Richardson R, Yablon SA, Sherer M. Prospective comparison of acute confusion severity with duration of post-traumatic amnesia in predicting employment outcome after traumatic brain injury. Journal of Neurology, Neurosurgery, and Psychiatry. 2007; 78(8):872-6.

7. Belanger HG, Vanderploeg RD. The neuropsychological impact of sports-related concussion: a meta-analysis. Journal of the International Neuropsychological Society. 2005; 11(4):345-57. [PubMed: 16209414]

8. McMahon P, Hricik A, Yue JK, et al. Symptomatology and Functional Outcome in Mild Traumatic Brain Injury: Results from the Prospective TRACK-TBI Study. Journal of Neurotrauma. 2013

9. NIH Concensus Development Panel on Rehabilitation of Persons with Traumatic Brain Injury. Rehabilitation of persons with traumatic brain injury. Journal of the American Medical Association. 1999; 282(10):974-83. [PubMed: 10485684]

10. Dikmen SS, Machamer JE, Winn HR, Temkin NR. Neuropsychological outcome at 1-year post head injury. Neuropsychology. 1995; 9(1):80.

11. Carroll L, Cassidy JD, Peloso P, et al. Prognosis for mild traumatic brain injury: results of the WHO collaborating centre task force on mild traumatic brain injury. Journal of Rehabilitation Medicine. 2004; 36(0):84-105. [PubMed: 15083873]

12. Frencham KA, Fox AM, Maybery MT. Neuropsychological studies of mild traumatic brain injury: A meta-analytic review of research since 1995. Journal of Clinical and Experimental Neuropsychology. 2005; 27(3):334-51. [PubMed: 15969356]

13. Schretlen DJ, Shapiro AM. A quantitative review of the effects of traumatic brain injury on cognitive functioning. International Review of Psychiatry. 2003; 15(4):341-9. [PubMed: 15276955]

14. Bigler ED, Farrer TJ, Pertab JL, et al. Reaffirmed Limitations of Meta-Analytic Methods in the Study of Mild Traumatic Brain Injury: A Response to Rohling et al. The Clinical Neuropsychologist. 2013; 27(2):176-214. [PubMed: 23356775]

15. Ruff, RM.; Cullum, CM.; Luerssen, TG. Neuropsychological Function and Brain Imaging: Springer. 1989. Brain imaging and neuropsychological outcome in traumatic brain injury; $\mathrm{p}$. 161-83.

16. Pagulayan KF, Temkin NR, Machamer JE, et al. The measurement and magnitude of awareness difficulties after traumatic brain injury: a longitudinal study. Journal of the International Neuropsychological Society. 2007; 13(4):561-70. [PubMed: 17521477] 
17. Ruff RM, Marshall L, Crouch J, et al. Predictors of outcome following severe head trauma: followup data from the traumatic coma data bank. Brain Injury. 1993; 7(2):101-11. [PubMed: 8453409]

18. Corrigan JD. Consequences of traumatic brain injury for functioning in the community. Rehabilitation of Persons With Traumatic Brain Injury. 1998; 51

19. Lezak, MD.; Howieson, DB.; Bigler, ED.; Tranel, D. Neuropsychological Assessment: OUP USA. 2012.

20. Spitz G, Ponsford JL, Rudzki D, Maller JJ. Association between cognitive performance and functional outcome following traumatic brain injury: a longitudinal multilevel examination. Neuropsychology. 2012; 26(5):604-12. [PubMed: 22799747]

21. Stuss DT. New approaches to prefrontal lobe testing. The Human Frontal Lobes: Functions and disorders. 2007; 2:292-305.

22. Dikmen SS, Corrigan JD, Levin HS, et al. Cognitive outcome following traumatic brain injury. The Journal of Head Trauma Rehabilitation. 2009; 24(6):430-8. [PubMed: 19940676]

23. Stuss DT, Alexander MP. Executive functions and the frontal lobes: a conceptual view. Psychological Research. 2000; 63:289-98. [PubMed: 11004882]

24. Shum D, Gill H, Banks M, et al. Planning ability following moderate to severe traumatic brain injury: Performance on a 4-disk version of the Tower of London. Brain Impairment. 2009; 10(03): 320-4.

25. Levin HS, Wilde E, Troyanskaya M, et al. Diffusion tensor imaging of mild to moderate blastrelated traumatic brain injury and its sequelae. Journal of Neurotrauma. 2010; 27(4):683-94. [PubMed: 20088647]

26. Bechara A, Damasio H, Damasio AR. Emotion, decision making and the orbitofrontal cortex. Cerebral Cortex. 2000; 10(3):295-307. [PubMed: 10731224]

27. Damasio, A. Descartes' error: emotion, reason, and the human brain GP Putnam's Sons. 1994. The somatic-marker hypothesis; p. 165-201.

28. Newcombe VF, Outtrim JG, Chatfield DA, et al. Parcellating the neuroanatomical basis of impaired decision-making in traumatic brain injury. Brain. 2011; 134(Pt 3):759-68. [PubMed: 21310727]

29. van Reekum R, Stuss DT, Ostrander L. Apathy: Why care? The Journal of Neuropsychiatry and Clinical Neurosciences. 2005; 17:7-19. [PubMed: 15746478]

30. Giancino JT, Cicerone KD. Varieties of deficit unawarness after brain injury. Journal of Head Trauma Rehabilitation. 1998; 13(5):1-15.

31. Sherer M, Boake C, Levin E, et al. Characteristics of impaired awareness after traumatic brain injury. Journal of the International Neuropsychological Society. 1998; 4:380-7. [PubMed: 9656611]

32. Flavell JH. Metacognition and cognitive monitoring: A new area of cognitive-developmental inquiry. Contemporary Readings in Child Psychology. 1981:165-9.

33. O'Keefe F, Dockree PM, Moloney P, et al. Awareness of deficits in traumatic brain injury: A multidimensional approach to assessing metacognitive knowledge and online-awareness. Journal of the International Neuropsychological Society. 2007; 13:38-49. [PubMed: 17166302]

34. Genarelli, T.; Grabau, D., editors. Neuropathology of head injures. Seminars in Clinical Neuropsychiatry. 1998.

35. Gennarelli TA, Thibault LE, Adams JH, et al. Diffuse axonal injury and traumatic coma in the primate. Annals of Neurology. 1982; 12(6):564-74. [PubMed: 7159060]

36. Smith DH, Meaney DF. Axonal damage in traumatic brain injury. The Neuroscientist. 2000; 6(6): 483-95.

37. Browne KD, Chen XH, Meaney DF, Smith DH. Mild Traumatic Brain Injury and Diffuse Axonal Injury in Swine. Journal of Neurotrauma. 2011; 28(9):1747-55. [PubMed: 21740133]

38. Wilde E, McCauley S, Hunter J, et al. Diffusion tensor imaging of acute mild traumatic brain injury in adolescents. Neurology. 2008; 70(12):948-55. [PubMed: 18347317]

39. Cicerone KD, Levin HS, Malec JF, et al. Cognitive rehabilitation interventions for executive function: Moving from bench to bedside in patients with traumatic brain injury. Journal of Cognitive Neuroscience. 2006; 18(7):1212-22. [PubMed: 16839293] 
40. Andersson S, Krogstad JM, Finest A. Apathy and depressed mood in acquired brain damage: relationship to lesion localization and psychophysiological reactivity Psychological Medicine. 1999; 29:447-56.

41. Spikman JM, van der Naalt J. Indices of impaired self-awareness in traumatic brain injury patients with focal frontal lesions and executive deficits: implications for outcome measurement. Journal of Neurotrauma. 2010; 27(7):1195-202. [PubMed: 20380551]

42. Schmitz TW, Rowley HA, Kawahara TN, Johnson SC. Neural correlates of self-evaluative accuracy after traumatic brain injury. Neuropsychologia. 2006; 44(5):762-73. [PubMed: 16154166]

43. Bigler ED, Bazarian JJ. Diffusion tensor imaging. Neurology. 2010; 74(8):626-7. [PubMed: 20107137]

44. Mayer A, Ling J, Mannell M, et al. A prospective diffusion tensor imaging study in mild traumatic brain injury. Neurology. 2010; 74(8):643-50. [PubMed: 20089939]

45. Niogi S, Mukherjee P, Ghajar J, et al. Extent of microstructural white matter injury in postconcussive syndrome correlates with impaired cognitive reaction time: a 3T diffusion tensor imaging study of mild traumatic brain injury. American Journal of Neuroradiology. 2008; 29(5): 967-73. [PubMed: 18272556]

46. Wilde EA, Whiteneck GG, Bogner J, et al. Recommendations for the use of common outcome measures in traumatic brain injury research. Arch Phys Med Rehabil. 2010; 91(11):1650-60. [PubMed: 21044708]

47. Hicks R, Giacino J, Harrison-Felix C, et al. Progress in developing common data elements for traumatic brain injury research: version two - the end of the beginning. Journal of Neurotrauma. 2013; 30(22):1852-61. [PubMed: 23725058]

48. Iverson GL. Outcome from mild traumatic brain injury. Current opinion in Psychiatry. 2005; 18(3): 301. [PubMed: 16639155]

49. Levine B, Schweizer TA, O'Connor C, et al. Rehabilitation of executive functioning in patients with frontal lobe brain damage with goal management training. Frontiers in Human Neuroscience. 2011; 5:9. [PubMed: 21369362]

50. Commitee on Cognitive Rehabilitation Therapy for Traumatic Brain Injury. Cognitive Rehabilitation Therapy for Traumatic Brain Injury: Evaluating the Evidence. Institute of Medicine; Washington, D.C.: 2011. 


\section{Synopsis}

Cognitive dysfunction is the leading cause of disability following traumatic brain injury (TBI). This article provides a review of the cognitive sequelae of TBI, with a focus on deficits of executive functioning and every day thinking skills. The pathophysiology, assessment, and treatment of TBI-related cognitive problems are also discussed. 


\section{Key Points}

- $\quad$ Cognitive deficits are a common consequence of acute traumatic brain injury (TBI), even at the lowest level of injury severity-concussion and mild TBI (mTBI). Approximately 65\% of moderate to severe TBI patients report longterm problems with cognitive functioning (1), and as many as $15 \%$ of mTBI patients have persistent problems, which often include cognitive deficits $(2$, $3)$.

- $\quad$ Deficit leads to disability when it interferes with patients' functional statusi.e. their ability to perform their usual pre-injury activities. Cognitive dysfunction is the leading cause of TBI-related disability, which affects approximately $43 \%$ of moderate to severe patients (4).

- $\quad$ Frontal lobe regions and their related circuitry are particularly vulnerable to TBI pathophysiology; and hence, executive deficits are common (5).

- Evaluation of cognitive functions should be routine in the assessment and treatment of post-acute TBI. Referral to a neuropsychologist can provide a thorough profile of an individual patient's cognitive strengths and weaknesses, which may aid in setting goals for treatment, career and education planning, or provide information about independent functioning.

- $\quad$ Cognitive rehabilitation therapy (CRT) is a safe treatment option for TBI patients with cognitive deficits; however, more methodologically rigorous research is needed to demonstrate its efficacy and identify which patients are most likely to respond. 
Table 1

Acute and Long-term Cognitive Sequelae of TBI by Levels of Severity.

\begin{tabular}{|c|c|c|}
\hline & Mild TBI & Moderate-Severe TBI \\
\hline \multicolumn{3}{|l|}{ Acute } \\
\hline Loss of consciousness & $0-30 \mathrm{~min}$ & $>30 \min$ \\
\hline Posttraumatic Amnesia & $0-24 \mathrm{hrs}$ & $>24 \mathrm{hrs}$ \\
\hline \multicolumn{3}{|l|}{ Subacute and Long-term } \\
\hline Memory & \multirow{4}{*}{$\begin{array}{l}\text { - Resolves rapidly within } 80-85 \% \text { patients } \\
\text { ( } 7,11-13 \text { ) } \\
\text { May persist in } ~ 15 \% \text { of patients (14) }\end{array}$} & \multirow{4}{*}{$\begin{array}{l}\text { - Persists in } \sim 65 \% \text { of patients (9) } \\
\text { - Can include deficits of awareness, reasoning, language, } \\
\text { visuospatial processing, and general intelligence (15-17) }\end{array}$} \\
\hline Attention & & \\
\hline Processing Speed & & \\
\hline Executive Functions & & \\
\hline
\end{tabular}


Table 2

Standard Neuropsychological Test Battery to Assess Cognitive Functioning Following TBI*

\begin{tabular}{l} 
Memory \\
Rey Auditory Verbal Learning (CDE)/ California Verbal Learning Test- II (CDE) \\
Brief Visuospatial Memory Test-Revised (CDE) \\
Processing Speed \\
Wechsler Adult Intelligence Scale-IV Processing Speed Index (CDE) \\
\hline Executive Functioning and Decision Making \\
Controlled Oral Word Association (CDE) \\
Trail Making Test (Trails A and B) (CDE) \\
Color Word Interference (CDE) \\
Iowa Gambling Task \\
Everyday Executive Functioning \\
Behavioral Report Inventory of Executive Functioning - Adult \\
Symptom Validity Assessment \\
Test of Memory Malingering (TOMM) (CDE)
\end{tabular}

${ }^{*} \mathrm{CDE}=$ Common Data Element 